\title{
Numerical Simulation of Heat Transfer on BFS during the Flight
}

\author{
Shun Li , Gongduo Zhang, Hui Jiang , Guowei Xie \\ Sinosteel Anshan Research Institute of Thermo-energy Co., LTD., Anshan, Liaoning, \\ 114044 ,China \\ asrnls2008@126.com
}

Keywords: standard coal, initial temperature, nucleation, crystal growth .

Abstract. At present, the domestic treatments of BFS are water-quenching processes. The existed studies on the centrifugal granulation were focused on slag particle shape, size and mass distribution. Slag temperature during the flight has not been reported. The relations between slag temperature and air temperature, initial temperature, rotary speed and slag diameter were studied. The results can provide reference for the design of sensible heat recovery device.

\section{Introduction}

Molten blast furnace slag is a by-product of blast furnace ironmaking, its exhaust temperature is $1400 \sim 1600^{\circ} \mathrm{C}$, enthalpy of about $60 \mathrm{Kg}$ standard coal per ton of slag. At present, the domestic treatments of BFS are water-quenching processes [1].

Centrifugal Granulation belongs to dry granulation, which granulates slag into drops by the rotary cup, and cools the drops by air and water and recovers slag sensible heat.

The existed studies on the centrifugal granulation were focused on slag particle shape, size and mass distribution [2 3], and slag temperature during the flight has not been reported. The relations between slag temperature and air temperature, initial temperature, rotary speed and slag diameter were studied. The results can provide reference for the design of sensible heat recovery device.

\section{Model Mathematical Formulation}

During the flight, molten slag transfers heat to the environment in radiation and convection. Slag temperature decreases gradually. Molten slag experiences three stages: liquid cooling, solidification process and solid cooling.

\section{Liquid Cooling}

At this stage, the heat loss of molten slag (convection and radiation) is compensated for the enthalpy. Based on the principle of heat transfer, molten slag temperature can be expressed by

$$
\frac{d T_{d}}{d t}=-\frac{6\left\lfloor h_{c}\left(T_{d}-T_{g}\right)+\sigma \varepsilon\left(T_{d}^{4}-T_{g}{ }^{4}\right)\right\rfloor}{\rho C_{p l} d_{p}}
$$

Where $T_{p}$ and $T_{g}$ respectively is temperature of molten slag and gas, $\sigma$ is Stefan-Boltzmann constant, $\varepsilon$ is molten slag blackness, $C_{p l}$ is specific heat for liquid, $h_{c}$ is convection heat transfer coefficient.

\section{Slag Solidification Process}

Slag solidification is a non-equilibrium process. From the dynamic point of view slag solidification process can be divided into two stages: nucleation and growth. However, the actual two processes are not completely separated. The JMA equation and the JMAK equation are widely used in the phase transformation kinetics.

Nucleation. The nucleation rate is expressed as 


$$
I=\frac{N_{0} k T}{3 \pi \eta a^{3}} \exp \left(-b \cdot \frac{a^{3} H_{m}}{R T_{m} \Delta T_{r}^{2} T_{r}}\right)
$$

Where $\mathrm{I}$ is nucleation rate, $\mathrm{a}$ is atom distance, $\mathrm{N} 0$ is atomicity of unit volume, $\mathrm{N} 0=1 / \mathrm{a}^{3}, \mathrm{k}$ is disposal of boltzmann's constant, $\mathrm{T}$ is absolute temperature, $\eta$ is viscosity, $\mathrm{b}$ is geometric factor, $b=16 \pi / 3$, $\mathrm{Hm}$ is the molar heat of fusion, $T_{r}=T / T_{m}$ reduced temperature), $\Delta T_{r}=1-T_{r}$ (reduced undercooling).

Crystal Growth. The growth speed on crystal mostly is described as

$$
U=\frac{f k T}{3 \pi \eta a^{2}} \cdot\left[1-\exp \left(-\frac{H_{m}}{R T_{m} \Delta T_{r}^{2} T_{r}}\right)\right]
$$

Overall Crystallization Rate. The overall crystallization rate is represented for between fraction of crystallized crystal volume accounted for the original liquid volume and crystallization time.

$$
x=\frac{V^{S}}{V}=\frac{4 \pi}{3} \int_{x}^{t} I U^{3}(t-\tau)^{3} d t
$$

When $\tau$ is very small, $\tau \rightarrow 0$, I and $U$ do not change with time

$$
x=\frac{\pi}{3} I U^{3} t^{4}
$$

When the nucleation rate is independent of crystal formation rate and time, and $t>>\tau$, corrected factor $1-\mathrm{x}$ on the collision between particles and the crystallization mother liquor in the process is obtained

$$
x=1-\exp \left(-\frac{\pi}{3} I U^{3} t^{4}\right)
$$

Heat Transfer in Crystallization Process. The slag is solid and liquid mixture, the latent heat of solidification makes up for heat loss in the nucleation and grain growth period, slag temperature should satisfy as [4]

$$
\frac{d T_{d}}{d t}=\frac{L d x}{C_{p l s} d t}-\frac{6\left[h_{c}\left(T_{d}-T_{g}\right)+\sigma \varepsilon\left(T_{d}{ }^{4}-T_{g}{ }^{4}\right)\right]}{\rho C_{p l s} d_{p}}
$$

\section{Solid Phase Cooling}

At this stage, the slag has turned completely solid, slag temperature is abide by

$$
\frac{d T_{d}}{d t}=-\frac{6\left[h_{c}\left(T_{d}-T_{g}\right)+\sigma \varepsilon\left(T_{d}{ }^{4}-T_{g}{ }^{4}\right)\right]}{\rho C_{p s} d_{p}}
$$

\section{Effect of Slag Diameter on Slag Temperature}

Under the conditions of different diameter of $1.5 \mathrm{~mm}, 2 \mathrm{~mm}, 2.5 \mathrm{~mm}$ and $3 \mathrm{~mm}$ the relations between the surface temperature of slag and time during the flight are shown in Figure 1.It is found that the smaller diameter is, the shorter solidification time is, the more quickly the slag temperature decreases.

For example $1.5 \mathrm{~mm}$ slag, because of the small size and the internal resistance the latent heat of solidification can quick release, the slag particle temperature drops rapidly. With the increase of slag diameter, its internal resistance increases gradually, solidification latent heat release requires more time gradually, the entire decrease of slag temperature slows down. In the future engineering production, in order to obtain completely solidified or partially solidified slag in a relatively short time, slag diameter can be small. But if slag diameter is too small, the cooling rate is too fast, which temperature has been low for waste heat recovery system .Therefore, in consideration of the system design, the corresponding relations exist between the proper time of flight and cinder diameter. 


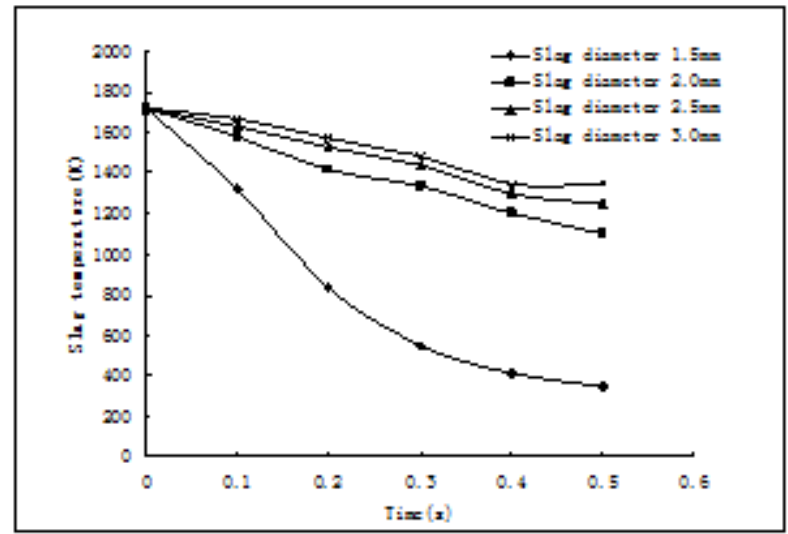

Figure-1 Relationship between slag diameter and slag temperature

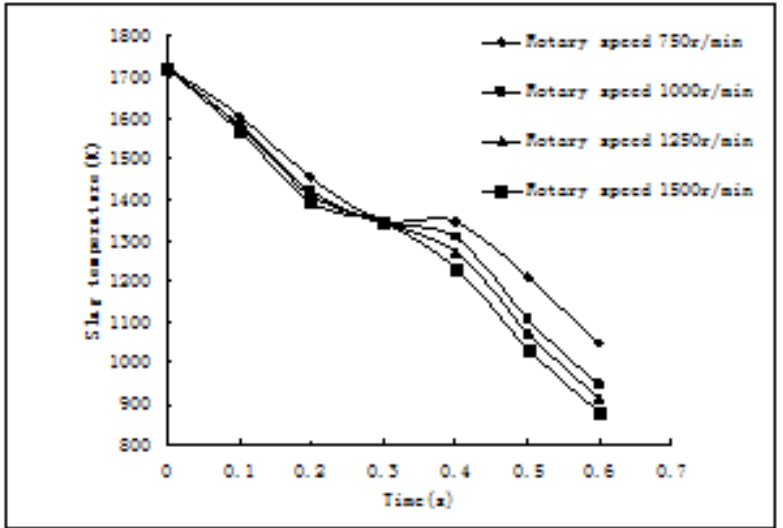

Figure 2 Relationship between rotary speed and slag temperature

\section{Effect of Rotary Speed on Slag Temperature}

Under the conditions of different rotary speed of 750rpm,1000rpm,1250rpm and 1500rpm the relations between the surface temperature of slag particles and time during the flight are shown in Figure 2.It is found that the surface temperature of slag gradually decreases with the increase of rotary speed gradually, especially after $0.3 \mathrm{~s}$ temperature decreasing rate increases significantly.

With the increase of rotary speed air disturbance around slag increases the convective heat transfer coefficient between the slag and the air increases, the total heat transfer coefficient increases, slag surface temperature gradually decreases. Before $0.3 \mathrm{~s}$ temperature decreasing rate is about $1000 \mathrm{~K} / \mathrm{s}$.After $0.3 \mathrm{~s}$ it reaches to $1700 \mathrm{~K} / \mathrm{s}$.

\section{Effect of Air Temperature on Slag Temperature}

Under the conditions of air temperature of $293 \mathrm{~K}$ and $393 \mathrm{~K}$ the relations between the surface temperature of slag particles and time during the flight are shown in Figure 3.It is found that the surface temperature of slag gradually decreases with the decrease of air temperature.

According to the formula the heat transfer rate is equal to the product of heat transfer coefficient and temperature difference. Under the conditions of the same heat transfer coefficient the greater the temperature difference is, the greater the heat transfer rate is. Improving air temperature means that the temperature difference reduces, which makes heat transfer rate decrease, so the temperature of slag increases. But the air temperature increases or decreases $100 \mathrm{~K}$, slag temperature decreases or increases $40 \mathrm{~K}$. Although air temperature has little effect on the slag temperature change, but it can shorten the time and reduce the slag solidification equipment size by reducing the air temperature.

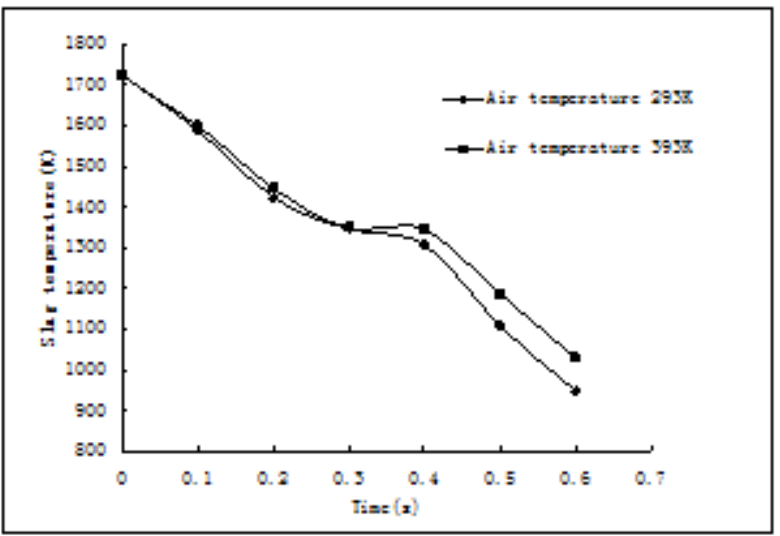

Figure-3 Relationship between air temperature and slag temperature

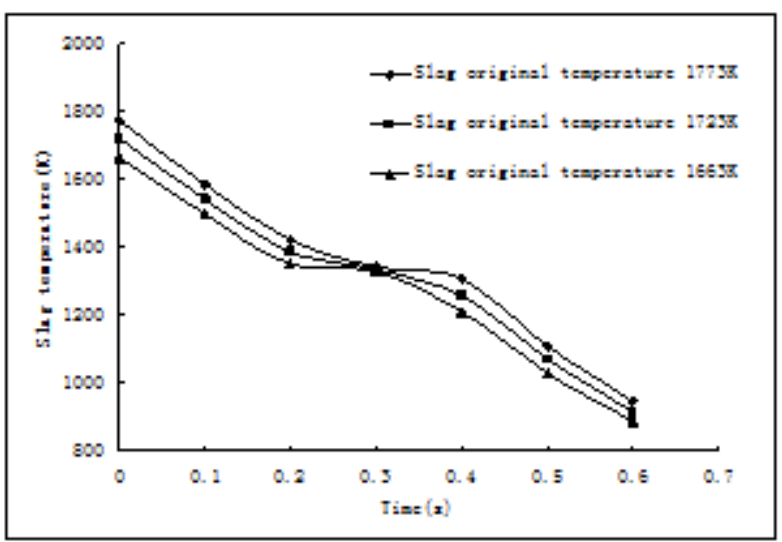

Figure-4 Relationship between initial temperature and slag temperature 


\section{Effect of Initial Temperature on Slag Temperature}

Under the conditions of different temperature of $1663 \mathrm{~K}, 1723 \mathrm{~K}$ and $1773 \mathrm{~K}$ the relations between the surface temperature of slag particles and time during the flight are shown in Figure 4.It is found that the surface temperature of slag gradually increases with the increase of the initial temperature.

According to the formula that heat transfer rate is equal to the product of the heat transfer coefficient and the temperature difference. Under the conditions of the same heat transfer coefficient, the greater the heat transfer rate is, the greater the temperature difference is. Improving the initial temperature of the slag means to provide the system with the more heat, which increases the temperature difference. Therefore, the surface temperature of slag increases. The greater the surface temperature of slag is, the slower solidification process is, which time is longer, the greater the need for equipment is. In engineering practice, under the premise of ensuring the quality of waste heat recovery, the initial temperature of slag should be reduced.

\section{Conclusions}

(1) The smaller diameter is, the shorter solidification time is, the more quickly the slag temperature decreases.

(2) The surface temperature of slag gradually decreases with the increase of rotary speed gradually, especially after $0.3 \mathrm{~s}$ temperature decreasing rate increases significantly.

(3) The surface temperature of slag gradually increases with the increase of air temperature or the increase of the initial temperature.

\section{Reference}

[1] WANG Hai-Feng, ZHANG Chun-Xia, QI Yuan-Hong: China Metallurgy Vol.17 (2007), p.53.

[2] Pickering S J, Hay N, Roylance T F ect:.Ironmaking and Steelmaking Vol.12 (1985), p.14.

[3] Mizuochi T, Akiyama T, Shimade T:ISIJ International Vol.41 (2001), p.1423.

[4] Clyne T W:Metall Trans B Vol.15 (1984), p.369. 